
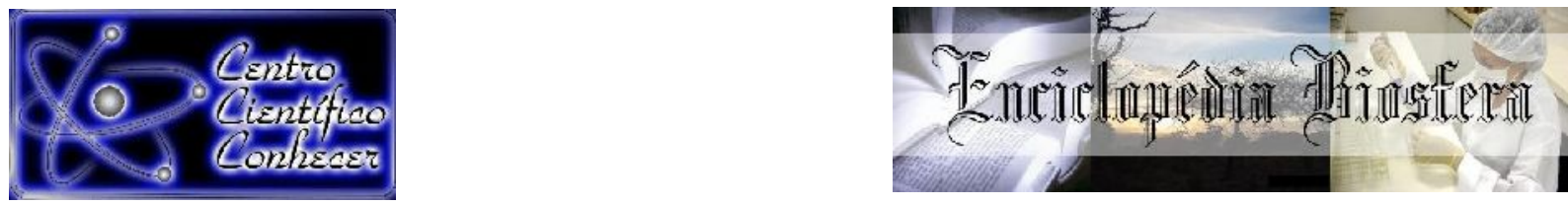

\title{
A PRODUÇÃO AGROINDUSTRIAL FRENTE ÀS PERSPECTIVAS BIOECONÔMICAS E DE DESENVOLVIMENTO SUSTENTÁVEL ATUAIS
}

\author{
Héberly Fernandes Braga ${ }^{1}$, Heloiza Ferreira Alves do Prado ${ }^{2}$ \\ ${ }^{1}$ Doutorando em Microbiologia - UNESP, Campus de São José do Rio Preto, São José \\ do Rio Preto - SP, Brasil. \\ ${ }^{2}$ Professora Doutora - UNESP, Departamento de Fitotecnia, Tecnologia de Alimentos e \\ Sócio Economia, Faculdade de Engenharia, Campus de Ilha Solteira, Ilha Solteira - SP, \\ Brasil \\ E-mail: heberly@iftm.edu.br
}

Recebido em: 15/05/2021 - Aprovado em: 15/06/2021 - Publicado em: 30/06/2021

DOI: 10.18677/EnciBio_2021B4

\begin{abstract}
RESUMO
A sociedade atual tem buscado alternativas que possam vincular o tão almejado crescimento e desenvolvimento, com padrões produtivos e de consumo menos agressivos à população, qualidade de vida e meio ambiente. Dentre essas alternativas, a adoção e inserção de modelos baseados em sustentabilidade e bioeconomia têm tido adesão de diferentes setores da sociedade, em especial da cadeia agroindustrial. Nesse sentido, o presente artigo de revisão objetivou abordar o atual cenário de inserção do setor agroprodutivo industrial nos moldes do desenvolvimento bioeconômico e sustentável, apresentando contribuições e apontamentos relativos às perspectivas, oportunidades e desafios dessa adoção, especialmente por países que têm como base econômica a produção primária. Nota-se que vários são os desafios e esforços necessários a essa inserção, mas também muitas são as oportunidades que podem ser obtidas, como agregação de valor à cadeia produtiva, e maior dinamicidade e visibilidade do setor, especialmente porque as ações e atividades baseiam-se no aproveitamento mais otimizado dos recursos.
\end{abstract}

PALAVRAS-CHAVE: Bioeconomia; Cadeia agroindustrial; Sustentabilidade.

\section{AGRO-INDUSTRIAL PRODUCTION IN THE FACE OF CURRENT BIOECONOMIC AND SUSTAINABLE DEVELOPMENT PROSPECTS}

\begin{abstract}
Today's society has been looking for alternatives that can link the longed-for growth and development, with less aggressive production and consumption patterns for the population, quality of life and the environment. Among these alternatives, the adoption and insertion of models based on sustainability and the bioeconomy have been adhered to by different sectors of society, especially in the agro-industrial chain. In this sense, this review article aimed to address the current scenario of insertion of the industrial agroproductive sector along the lines of bioeconomic and sustainable development,
\end{abstract}


presenting contributions and notes regarding the prospects, opportunities and challenges of this adoption, especially by countries whose economic base primary production. It is noted that there are several challenges and efforts necessary for this insertion, but also many opportunities that can be obtained, such as adding value to the production chain, and greater dynamism and visibility in the sector, especially because the actions and activities are based on the most optimized use of resources.

KEYWORDS: Bioeconomy; Agro-industrial chain; Sustainability.

\section{INTRODUÇÃO}

O cenário global da sociedade contemporânea marcado em grande parte pelo sistema capitalista, que vem ao longo dos tempos estimulando a produção e consumo desenfreados tem demandado por recursos naturais, o que dispõe no ambiente grande quantidade de rejeitos e energia, que devido a sua constituição e/ou alta carga emitida, demoram a ser transformados e reutilizados naturalmente. Tal situação gera desordens ambientais que podem promover distintos problemas sejam de caráter social, econômico e/ou ambiental (MIRANDA, 2018).

Essas desordens iniciadas, em particular no século XVIII, decorrentes da Revolução Industrial e acentuadas nos anos seguintes, especialmente a partir do século XX com o advento da globalização, têm suscitado na humanidade um clamor por soluções que visem congregar o paradoxo do desenvolvimento social e econômico associado à conservação e proteção da vida e do ambiente, de modo a dar um direcionamento mais sustentável à sociedade (ONU, 2015; LAMPKOWSKI; LAMPKOWSKI, 2016; MIRANDA, 2018). Assim, a busca por processos mais sustentáveis nos diferentes setores, e em específico na agropecuária e cadeia agroindustrial, se insere como parte importante dos modelos de gerenciamento e manejo produtivo e de negócios (SILVA, 2012).

Nesse contexto, a presente revisão objetivou trazer algumas contribuições do cenário que apontam os possíveis desafios, perspectivas e oportunidades de inserção da cadeia agroindustrial nesse novo sistema de desenvolvimento pautado na sustentabilidade e bioeconomia.

\section{O CENÁRIO DE INSERÇÃO DO SETOR AGROINDUSTRIAL NO ATUAL MODELO DE DESENVOLVIMENTO BIOECONÔMICO E SUSTENTÁVEL}

Os desafios econômicos impostos às sociedades atuais estão atrelados a diferentes fatores sejam eles ambientais, sociais ou financeiros, que podem levar a instabilidade de setores produtivos individuais ou mesmo de economias inteiras. A interconexão global existente na natureza e mesmo aquela realizada pelo homem por meio da globalização, trazem benefícios como expansão de mercados, acesso à informação e tecnologia, entre outros; mas também podem incorrer em desvantagens como empobrecimento de uma grande parcela da sociedade, problemas ambientais e de saúde pública, como por exemplo a atual pandemia da Síndrome Respiratória Aguda Grave de Coronavírus 2 (SARS-CoV-2) (CAVALCANTE, 2016; FERREIRA, 2017; GEISSDOERFER et al., 2017; DINIZ et al., 2020; SOFFIATI, 2020).

Para Alencar et al. (2019), a temática do desenvolvimento associado à globalização e sustentabilidade, é uma discussão de cunho social e científico, pois 
aborda assuntos globais, além do que, é um direito fundamental e consagrado para que as gerações atuais e futuras de uma sociedade possam suprir suas necessidades. Segundo Soffiati (2020), o entendimento da atual crise socioambiental é inerente ao modo produtivo capitalista, mas decorre historicamente dos vários modos de interação do homem com a natureza ao longo dos tempos.

Historicamente, as desordens ambientais derivadas da modificação dos modos de produção e consumo, devidos à revolução industrial, intensificaram a busca por recursos naturais em nome do progresso e desenvolvimento econômico, levando a degradação em larga escala da natureza. Tais eventos foram ainda mais exacerbados com o advento da globalização, o que gerou e tem gerado distintos conflitos e propostas para se mitigá-los (BARROS, 2020).

O panorama mundial das pesquisas tem mostrado que uma das formas de mitigar as crises econômico-sócio-ambientais da atualidade perpassa pela inserção e imersão dos países e seus sistemas políticos, em projetos, programas e ações associados ao desenvolvimento sustentável (THORSTENSEN; MOTA, 2018; ALENCAR et al., 2019; BARROS, 2020; SILVA et al., 2020a), exigindo uma observação de todas as facetas dos problemas e o engajamento de todos os atores sociais, incluindo governos, sociedade civil, organizações não-governamentais, setor privado, industrial e de pesquisa (GEISSDOERFER et al., 2017; BARROS, 2020). Essa mobilização de diferentes agentes, atrelada ao investimento e desenvolvimento local, podem também reduzir as disparidades e garantir a longo prazo uma melhor sustentabilidade (FERREIRA, 2017).

Segundo Sarlet (2017), as questões relativas ao desenvolvimento sustentável conglomeram distintos aspectos da vida em sociedade, assim como da interação entre os países, apresentando um caráter dinâmico e mutável. A modificação dos interesses e necessidades humanas, alteram o modo de se pensar e agir em termos sustentáveis. As inquietações entre os objetivos da conservação ambiental e o desenvolvimento socioeconômico, clamam por uma visão integrada que articulem ambas as facetas, propiciando ao mesmo tempo o crescimento, mas também a manutenção, conservação e renovação ambiental.

O Brasil é um País que tem uma forte base agropecuária, seja devido à sua extensão territorial, abundância fluvial, ou mesmo às condições do solo e clima, que propiciam a produção de uma variedade de espécies agrícolas, como cana-de-açúcar, mandioca, laranja, soja, milho, entre outras; além da criação e desenvolvimento de animais para abate, ou como fornecedores de leite, ovos ou mel. Essas atividades têm importância econômica e social, pois geram empregos diretos e indiretos e distribuem renda à população. Entretanto, a produção e beneficiamento de produtos agropecuários está diretamente associada à geração de resíduos e subprodutos, que se não forem adequadamente geridos podem promover impactos socioambientais negativos, comprometendo a qualidade de vida da sociedade e a sustentabilidade e desempenho das atividades econômicas (CORDEIRO et al., 2020).

Em termos de desempenho ambiental, o Brasil apresentou nos últimos anos, queda no ranking (Environmental Performance Index - EPI) passando da 46 em 2016 (HSU et al., 2016) para a 69aำ posição em 2018 (EPI, 2018). Tais indicadores do EPI medem a proximidade do país ao cumprimento de metas estabelecidas 
internacionalmente quanto à proteção da saúde humana e proteção dos ecossistemas. No que tange ao setor agropecuário e agroindustrial, o volume agroprodutivo exerce impactos significativos na sustentabilidade do país (MARQUES et al., 2019), especialmente se não for adequadamente manejado. Conforme relatório emitido pelo Instituto Brasileiro de Geografia e Estatística - IBGE, a agricultura foi uma das atividades humanas que mais demandou água em 2017, por exemplo (IBGE, 2017).

Um outro fator que influi no desempenho ambiental do setor agropecuário e agroindustrial brasileiro é a grande quantidade de rejeitos gerados anualmente e provenientes de diferentes culturas, atividades e processos. Conforme Moraes et al. (2017) é estimado que a quantidade de biomassa produzida no Brasil possa atingir aproximadamente $1 \mathrm{Gt}$ em 2030. Conforme os mesmos autores as sobras geradas, incluindo também aquelas do processamento, ainda são subutilizadas, sendo dispostas de forma inadequada no solo, gerando além de desperdícios de nutrientes e energia, passivos ambientais de significativo impacto.

O bagaço da laranja, se disposto inadequadamente no solo, pode promover danos ambientais em virtude da lenta decomposição decorrente da sua constituição lignocelulósica (SANTIAGO; RODRIGUES, 2017). Já, os subprodutos da mandioca (casca marrom, entrecasca, fibra, bagaço, entre outros), assim como a água de lavagem derivada da obtenção da fécula, contém particulados e ácido cianídrico, que podem contaminar o solo, lençol freático, e rios, promovendo, por exemplo, a mortandade de peixes devido à maior demanda bioquímica do oxigênio no corpo d'água. Um dos principais derivados do beneficiamento da mandioca para a produção de fécula é o farelo, e estima-se em média que para cada $1000 \mathrm{~kg}$ de raiz processada, gera-se em torno $930 \mathrm{~kg}$ desse farelo (MARTINEZ; FEIDEN, 2017).

Nascimento Filho e Franco (2015) relatam que as sobras e resíduos gerados são provenientes de diversas etapas da cadeia produtiva, havendo estimativas de que somente cerca de $85 \%$ da biomassa vegetal seja realmente aproveitada e $30 \%$ rejeitada. Dentre os diferentes motivos associados a tal desperdício estão a grande oferta de matéria prima vegetal disponível no País; o desenvolvimento ainda incipiente e pouco difundido de tecnologias de aproveitamento; a pseudo impressão empresarial de que os subprodutos são considerados custos operacionais adicionais à empresa/indústria; o baixo investimento em pesquisas sobre o assunto; e políticas governamentais internas que estimulem esse setor.

Devido à riqueza em nutrientes e energia apresentada por muitos dos subprodutos (casca, bagaço, polpa, sementes, caroços, folhas, talos e hastes) da biomassa vegetal (SARAIVA et al., 2018), vários estudos têm ganhado notoriedade e investimentos recentes em diferentes países, incluindo até mesmo aqueles com alto desenvolvimento tecnológico, como a França e Estados Unidos (NASCIMENTO FILHO; FRANCO, 2015). Os subprodutos têm sido aproveitados como coprodutos para elaboração de alimentos (SANTOS et al., 2018); produtos, utensílios e serviços humanos (DIAS; REIS, 2019; BELTRAN et al., 2020; SILVA et al., 2020b); como ração para animais de criação (CARVALHO et al., 2016); adubo e fertilizantes (CRUZ et al., 2017); na produção de biocombustível e elaboração fermentativa de bioprodutos (PENHA et al., 2016; BARROS et al., 2019); entre outros, possibilitando agregar valor à cadeia produtiva (NWACHUKWU et al., 2016). 
As discussões científicas sobre a adoção de práticas sustentáveis no setor agropecuário e agroindustrial vêm crescendo nos últimos anos, em diversas atividades e regiões do Brasil, como comprova um estudo exploratório de levantamento da produção científica brasileira sobre o assunto realizada por Zanella e Lago (2016), em periódicos nacionais, com estratos de qualificação Qualis Capes A1 a B3, publicados entre 2005 e 2015. De acordo com as autoras, em geral a adoção de ações sustentáveis é incorporada em decorrência de exigências legais, ou necessidade da obtenção de certificações, mas algumas organizações produtivas já utilizam tais práticas com um caráter estratégico em suas metas mercadológicas e tecnológicas, transformando-as em oportunidades de negócios.

A sustentabilidade nos mais variados segmentos econômicos tem se tornado cada vez mais necessária visando um desenvolvimento ecologicamente correto, que proporcione melhor qualidade de vida à sociedade sem deixar a lucratividade financeira dos negócios. A sociedade vem se conscientizando a cada dia da necessidade de adotar alternativas e estratégias sustentáveis, que possam ao mesmo tempo elevar os níveis produtivos e otimizar o uso dos recursos naturais, de modo a atender as necessidades da geração atual, sem esquecer das gerações futuras e promover danos irreparáveis ao meio ambiente (SILVA, 2012; TORRES et al., 2014; SILVA; MEGALE, 2020).

De acordo com Barros (2020), a visão biocêntrica do mundo em contraposição à visão antropocêntrica vem ganhando gradativamente espaço, haja vista a crise ambiental estabelecida. Para a autora é mais que um dever da sociedade repensar sobre o caminho de exploração da natureza percorrido pela humanidade, e adotar atitudes conjuntas entre cidadãos e governo, que propiciem um ambiente ecologicamente equilibrado e um desenvolvimento economicamente sustentável.

Nesse sentido, a bioeconomia tem se mostrado como uma solução plausível para que a escassez de recursos naturais se equilibre frente ao consumo, e a produção dos bens, os quais dependem desses recursos (LAMPKOWSKI; LAMPKOWSKI, 2016), podendo oferecer soluções coerentes e concretas a esses desafios ambientais impostos à sociedade (BARROS, 2020). Para Moreira (2016), o conceito de bioeconomia sempre esteve inserido no setor agropecuário e agroindustrial, mas só recentemente vem sendo abordado de maneira mais estratégica.

Conforme Moretti (2020), investimentos em pesquisa agropecuária e agroindustrial no Brasil podem trazer retornos à sociedade brasileira, e no âmbito da Empresa Brasileira de Pesquisa Agropecuária (EMBRAPA), temas como intensificação sustentável e economia de base biológica (bioeconomia) se destacam no momento atual e futuro. Além disso, o uso adequado da biodiversidade pelo emprego da tecnologia tem papel fundamental na sustentabilidade, ao propiciar o reaproveitamento dos materiais, combatendo o desperdício e a poluição devida ao descarte indevido dos rejeitos. Outro ponto importante relaciona-se à contribuição que essas atividades podem trazer para o impulsionamento de inovações no mercado associadas ao ciclo natural e o desenvolvimento econômico comunitário (BARROS, 2020).

Estima-se que até 2030 a população global cresça 16\% saindo dos 7,3 bilhões de habitantes em 2015, para 8,5 bilhões (UNITED NATIONS, 2017). Projeções indicam que a extração de recursos da natureza pode aumentar 119\% de 2015 para 2050, com 
um crescimento nas emissões dos gases estufa de até 41\% (HATFIELD-DODDS et al., 2017). Aliado a esse aumento populacional, demandas e desafios que possam agregar o desenvolvimento pautado em um modelo que permita reduzir a poluição, conservar e otimizar o uso dos recursos naturais e preservar a biodiversidade têm sido buscados.

Para Aquilani et al. (2018) é preciso que os países adotem sistemas econômicos mais sustentáveis, capazes de garantir a produção e consumo mais limpos e seguros, com base em inovação e tecnologia, reduzindo, reciclando e reutilizando os recursos. Para isso, uma das alternativas de materializar esse novo paradigma seria abrir-se ao modelo de desenvolvimento pautado na Bioeconomia (EL-CHICHAKLI et al., 2016; IPEA, 2017).

Conforme Silva et al. (2018), não há um consenso sobre a definição conceitual de bioeconomia, pois esta tem evoluído e variado conforme os autores e países, entretanto existem elementos convergentes entre as diferentes definições, que são: as origens em atividades primárias; enfoque transversal; e ênfase na produção com emprego de tecnologias avançadas de conversão de produtos. Para os autores a produção e comercialização de produtos têxteis naturais, bem como, cervejas, vinhos e queijos foram os primeiros indícios tradicionais, que têm sido aprimorados com o tempo, e vêm constituindo a bioeconomia moderna.

A Organisation for Economic Co-operation and Development - OECD, definiu o termo bioeconomia, como uma atividade que a biotecnologia está atrelada à produção econômica, envolvendo desenvolvimento sustentável, biomassa renovável e integração e aplicação desses setores (OECD, 2009). Membros do Conselho de Pesquisa em Tecnologia e Bioeconomia da Alemanha definiram essa atividade econômica como a área que engloba todos os setores e serviços que produzem, processam ou utilizam quaisquer recursos biológicos renováveis, independentemente de sua forma (HÜNTTL et al., 2011).

De acordo com a Comissão Europeia, a bioeconomia baseia-se na produção de alimentos, rações, produtos de base biológica e bioenergia, empregando-se recursos renováveis, bem como resíduos dos processos produtivos de transformação; tendo como viés constitutivo os bioprocessos de conversão de biomassa, a biotecnologia e os distintos produtos (vacinas, medicamentos, enzimas, etc.) por ela gerado, assim como os processos e serviços associados à sua aplicação na saúde, indústria e meio ambiente (EUROPEAN COMMISSION, 2012).

Para várias agências e departamentos governamentais dos Estados Unidos (DOE et al., 2016), a definição de bioeconomia tem por base a substituição de recursos fósseis por fontes de biomassa, assim como o uso de ferramentas da biotecnologia, que possam gerar energia e produtos sustentáveis, promovendo benefícios econômicosócio-ambientais e de segurança nacional. Segundo Parisi e Ronzon (2016), a bioeconomia no Canadá é definida como atividade associada à invenção, desenvolvimento e uso de processos e produtos baseados em recursos biológicos, incluindo diferentes setores da atividade humana.

$\mathrm{Na}$ França a bioeconomia foi definida como o conjunto de atividades associadas à produção, uso e transformação de biorrecursos de forma sustentável, visando atender as necessidades da sociedade no que tange a materiais, energia e alimentação (FRANÇA, 2017). 
Conforme a Confederação Nacional da Indústria (CNI) do Brasil, a bioeconomia é o resultado de uma revolução de inovações no campo das Ciências Biológicas, estando atrelada à invenção, desenvolvimento e uso de produtos e processos biológicos em diferentes setores da atividade humana, com destaque para a biotecnologia industrial (produção e processos químicos/enzimáticos; aplicações ambientais e biocombustíveis), produção primária (melhoramento de plantas e animais e aplicações veterinárias) e saúde humana (terapêutica, farmacologia, alimentos funcionais e equipamentos médicos) (CNI, 2013).

De acordo com Silva et al. (2018), as diferentes definições de bioeconomia empregam tanto processos produtivos baseados em conhecimentos tradicionais, quanto processos baseados no uso intensivo de novos conhecimentos científicos e tecnológicos no campo das Ciências Biológicas, sejam da biotecnologia moderna, genômica, biologia sintética, bioinformática e engenharia genética.

As bases da bioeconomia atual, que vem gradativamente sendo adotada em vários países, na elaboração de programas fundamentados no uso sustentável dos recursos biológicos, remontam aos eventos e sistemas de produção que marcaram o século XX, baseados na dependência de recursos não renováveis, tal como o petróleo, que acabaram por promover desordens e problemas socioambientais ao longo do tempo (BIBER-FREUDENBERGER et al., 2018). Nesse sentido, a bioeconomia surge como alternativa que visa transformar o conhecimento em desenvolvimento industrial sustentável, em áreas ligadas ao manejo e aproveitamento de recursos biológicos, visando integrar setores e solucionar déficits socioeconômicos (MOREIRA, 2016).

A manipulação sustentável da biodiversidade empregando-se tecnologia pode contribuir com o reaproveitamento de produtos e criação de novas fontes energéticas, que venham a combater o desperdício e poluição decorrentes da emissão ou descarte de rejeitos no ambiente. Além disso, a adoção dessas técnicas e comportamentos podem impulsionar atividades inovadoras alinhando o ciclo da natureza e o desenvolvimento econômico de uma sociedade (BARROS, 2020). Exemplo disso, deriva de um estudo realizado por Gonçalves Júnior et al. (2018), que verificaram potencial alternativa no uso do endocarpo de açaí como biossorvente de cádmio, chumbo em cromo, propiciando a recuperação de águas contaminadas por esses elementos químicos.

Sabe-se que o aspecto central para a propulsão da bioeconomia é uso e aplicação da biotecnologia em suas distintas variantes. Estudo prospectivo realizado pela OECD, em 2009, já estimava que até 2030, a contribuição global da biotecnologia poderá atingir 1 trilhão de dólares por ano, distribuídos no setor da saúde (US\$260 bilhões/ano), produção primária (US\$ 380 bilhões/ano) e industrial (US\$ 420 bilhões/ano) (OECD, 2009).

Segundo Barba e Santos (2020), os recursos da biodiversidade estão intimamente associados à biotecnologia, no que tange não somente às questões teóricas, como também práticas. Estudos biotecnológicos prospectam materiais, processos ou serviços da biodiversidade, que visam criar, transformar, modificar ou promover melhorias no meio em que se vive, seja em termos agropecuários, medicinais, industriais, alimentícios, entre outros. No sentido oposto, a biotecnologia pode gerar conhecimentos que propiciem a conservação e manutenção da 
biodiversidade, assim como valores de mercado à mesma, ao utilizar de informações, matéria-prima e conhecimentos tradicionais associados, que suscitam em diferentes bens e serviços.

De acordo com Moreira (2016), governos de países como Estados Unidos, Canadá, Alemanha, Rússia, Austrália, Grã-Bretanha e Finlândia já adotam programas estritamente alinhados a bioeconomia, direcionando políticas e incentivos ao uso de fontes não renováveis, de modo a estimular o crescimento econômico e dos empregos, mas ao mesmo tempo contribuir com soluções aos problemas ambientais. Para Morrison e Golden (2015), não somente o setor público tem reafirmado o compromisso com o uso de insumos biológicos, mas várias empresas do setor privado têm aderido a tal modelo econômico mais sustentável.

Ressalta-se a necessidade e importância que os países desenvolvidos têm em auxiliar e estimular os países subdesenvolvidos e/ou em desenvolvimento, a adotarem tais medidas, visando contribuir com a melhor qualidade ambiental e socioeconômica global. Em 2001 na Conferência das Nações Unidas sobre Comércio e Desenvolvimento já se pontuava a importância da maior participação dos países em desenvolvimento na bioeconomia, especialmente porque como tais países tem como base produtiva recursos naturais, eles podem beneficiar-se do uso da biotecnologia (JUMA; KONDE, 2001). Além disso, segundo Turine e Macedo (2017), faz-se importante também envolver as comunidades locais nesse cenário, pois os conhecimentos tradicionais sobre a biodiversidade local podem trazer respostas mais efetivas às problemáticas, e auxiliar na interação entre os agentes interessados envolvidos.

A bioeconomia no Brasil é bem exemplificada em projetos que envolvem o uso de enzimas e micro-organismos em processos fermentativos para produção de alimentos, aromas e ingredientes funcionais; produção de plantas com conteúdo proteico de alto valor biológico; redução de perdas em processos e distribuição, bem como no aproveitamento de subprodutos para o desenvolvimento de novas frações e de produtos de maior valor agregado (MOREIRA, 2016). Soma-se a isso, o desenvolvimento de plantas resistentes a diferentes climas; a produção de biofertilizantes; o controle de pragas empregando a seleção de micro-organismos naturais ou modificados geneticamente; e estudos relacionados a geração energias limpas empregando biocombustíveis (BARBA; SANTOS, 2020).

Para Dias e Carvalho (2017), o Brasil configura-se como um dos principais atores nesse novo cenário que envolve a bioeconomia, não somente porque possui uma enorme biodiversidade ainda pouco conhecida, e menores custos na produção de biomassa, como também aptidões agropecuárias e conhecimentos em processos agroindustriais relacionados especialmente à bioenergia. Além disso, são constatadas intensificações de iniciativas para se estruturar conselhos e plataformas conceituais que fomentem projetos de pesquisa e trabalhos envolvendo Bioeconomia (MOREIRA, 2016).

Dentre os diferentes estímulos de incentivo ao desenvolvimento nacional do setor da Bioeconomia, pode-se citar o Programa de Apoio à Inovação dos Setores Sucroenergético e Sucroquímico (PAISS), lançado em 2010 por uma iniciativa do Banco Nacional de Desenvolvimento Econômico e Social (BNDES) e da Financiadora 
de Estudos e Projetos (FINEP), que visava selecionar planos de negócio e fomento a projetos que tivessem como foco o desenvolvimento, produção e comercialização de novas tecnologias industriais destinadas ao processamento da biomassa oriunda principalmente da cana-de-açúcar (MOREIRA, 2016).

Em 2011, a CNI lançou uma agenda para estimular a inovação no Brasil, pautada entre outros temas, na biotecnologia e biodiversidade. No ano seguinte, em parceria com Harvard Business Review Brasil (HBR Brasil), foi realizado o "Fórum de Bioeconomia: desenvolvendo uma agenda para o Brasil". Este evento foi considerado o primeiro debate multissetorial e internacional sobre 0 assunto, demonstrando 0 interesse da inserção do país nesse novo formato de desenvolvimento econômico, social e ambiental. Em 2013, ocorreu o segundo fórum, o qual teve como produto um relatório que visou identificar as oportunidades e entraves, além de validar o potencial de expansão do segmento no país (CNI, 2013).

Outros eventos e ações de incentivos foram acontecendo ao longo dos anos, até mesmo em níveis da educação básica, tecnológica, superior e de pesquisa, podendo-se destacar mais recentemente, em 2019, a Semana Nacional de Ciência e Tecnologia (SNCT), com temática voltada a Bioeconomia (MCTIC, 2019).

Em 2013, uma pesquisa realizada pela HBR Brasil em parceria com a CNI conduziu o primeiro levantamento sobre a bioeconomia no Brasil, com 369 especialistas, executivos, interessados no assunto e pessoas que não tinham conhecimento sobre o tema. A pesquisa teve o objetivo de analisar o estágio de maturidade do país em relação não só ao entendimento e conhecimento sobre o assunto, como também o nível potencial de crescimento nacional nessa área. Na época foi verificado que $70 \%$ dos entrevistados mencionaram que o tema não fazia parte das discussões e iniciativas das instituições nas quais os mesmos estavam inseridos. Entretanto, os entrevistados mencionaram, dentre outros aspectos, nunca escala de 1 (pouco importante) a 5 (muito importante), que os conhecimentos advindos da biorrefinarias (42\%) e bioquímica (26\%) seriam de grande importância para contribuir com a Bioeconomia no Brasil nos cinco anos seguintes (CNI, 2013).

Já, Silva et al. (2018) verificaram em 2016, que as vendas no mundo, de produtos atribuíveis à bioeconomia brasileira, atingiram um valor em torno de US\$ 326,1 bilhões, sendo os setores da agropecuária, produtos alimentícios e bebidas, os que mais contribuíram nessa participação (73\%). É interessante ressaltar que os autores da pesquisa mencionam que os rejeitos do setor primário e urbano não foram considerados nas estimativas para contabilizar o valor da bioeconomia, mas que pesquisas futuras que incluam a atribuição dos mesmos seriam potencialmente importantes.

No âmbito do setor primário e de agronegócios o Brasil tem uma oportunidade ímpar de participar de maneira ainda mais significante na bioeconomia, haja visto ser o quinto maior país do mundo em extensão territorial; possuir $13,7 \%$ da reserva de água doce do planeta; estar localizado numa região tropical privilegiada com alta incidência solar e regime pluviométrico adequado (CNI, 2013). Associado a isso é importante se ressaltar, que o conhecimento vem se tornando um dos maiores bens econômicos da atualidade. $\mathrm{E}$ a biodiversidade nesse cenário tem papel primordial ao ofertar conhecimentos e aplicações que são ainda muito pouco estudados, especialmente em 
regiões de grade extensão territorial e diversidade, como o Brasil (BARBA; SANTOS, 2020).

Conforme Landau et al. (2020), a dinamicidade do setor agropecuário brasileiro pautado em aspectos e atividades bioeconômicas tem influências variadas sejam técnicas, econômicas, políticas, infra-estruturais, logísticas, históricas, culturais e ambientais, mas que independente da origem, geram demandas e oportunidades locais, nacionais e mesmo internacionais.

Empresas das áreas de agricultura, energia e meio ambiente que empregam biotecnologia e/ou processos biotecnológicos no Brasil, estão em franco crescimento. Para se ter ideia, uma pesquisa realizada em 2011, pelo Centro Brasileiro de Análise e Planejamento (CEBRAP) em parceria com a Associação Brasileira de Biotecnologia (BrBiotec), evidenciou que $25 \%$ das empresas dessas áreas (Figura 1), de um total de 237 mapeadas tinham a biotecnologia como atividade principal, e/ou desenvolviam projetos empregando-a (CEBRAP, 2011).

FIGURA 1. Percentual de empresas de biotecnologia no Brasil por área de atuação em 2011.

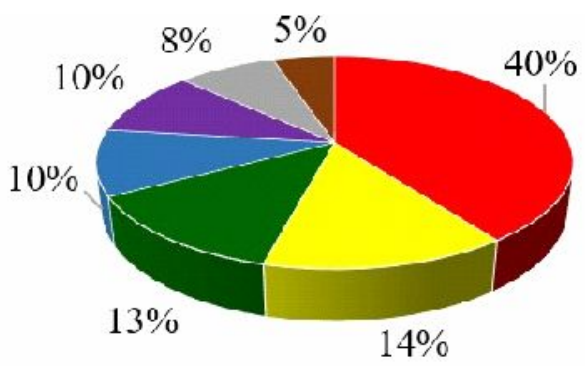

$\begin{array}{lll}\text { - Saúde humana } & \text { "Saúde animal } & \text { - Reagentes }\end{array}$

Fonte: CEBRAP (2011).

Um dos principais desafios do desenvolvimento da bioeconomia no Brasil perpassa pelo investimento em conhecimento, pesquisas, formação de recursos humanos e estruturação das biorrefinarias (SILVA et al., 2018). Para Barba e Santos (2020), faz-se necessário também estimular as parcerias entre universidades, institutos públicos de pesquisa e empresas; incentivar a transferência de conhecimentos e tecnologias, valorizando conhecimentos tradicionais e locais; além de se investir no arcabouço legal e executivo que possa assegurar a proteção ao uso e compartilhamento dos recursos derivados da biodiversidade.

Segundo Scarlat et al. (2015) as biorrefinarias são as unidades industriais potencialmente capazes de materializar uma desejada abordagem integrada e associada aos interesses almejados na Bioeconomia. Tais unidades baseiam-se na conversão da biomassa em bioenergia e bioprodutos, entretanto, na América do Sul, a maioria das biorrefinarias ainda são de primeira geração, trabalhando com açúcares 
provenientes da soja e da cana-de-açúcar, estando as plantas processadoras de resíduos agroindustriais ainda em fase inicial de implementação (NGUYEN et al., 2017; HASSAN et al., 2019).

Outro ponto a se destacar é a concentração de alguns setores bioeconômicos brasileiros em determinadas regiões geográficas específicas. Segundo Oliveira e Zanin (2015), a cadeia produtiva do etanol é bem organizada, mas ainda se encontra concentrada na região sudeste. Para os autores fazem-se necessários investimentos e incentivos socioeconômicos em regiões do norte e nordeste do Brasil, para que a produção de biocombustíveis, por exemplo, se torne mais competitiva, estratégica e sustentável. Trabalho realizado por Cavalcante Filho et al. (2019), frente à cadeia produtiva do biodiesel, também observou a mesma característica de concentração regional das atividades nas regiões centro-oeste e sul, e o foco especificamente direcionado à soja, como matéria-prima do processo produtivo. Para os autores políticas públicas que possam expandir a participação de outras oleaginosas, de modo a serem competitivas e compatíveis com as diferentes regiões produtivas brasileiras, são necessárias para promover o melhor desenvolvimento e dinâmica regional.

\section{CONSIDERAÇÕES FINAIS}

Apesar de muitos desafios e esforços necessários à consolidação de modelos de desenvolvimento pautados em sustentabilidade e bioeconomia, os apontamentos e pesquisas atuais mostram que a inserção atual da cadeia agroprodutiva industrial nesses sistemas são importantes e visam dar maior visibilidade, dinamizar e tornar mais estratégico o setor, especialmente em países que tem tais atividades como principal pilar de sustentação econômica. Nesse sentido, o fomento privado e principalmente governamental de iniciativas relacionadas ao assunto é muito importante para que possa alavancar e manter tais atividades tanto por pequenos, quanto médios e grandes produtores, e ao mesmo tempo em consonância com a conservação do meio ambiente tão almejada pela população global.

\section{AGRADECIMENTOS}

Ao Instituto Federal de Educação, Ciência e Tecnologia do Triângulo Mineiro (IFTM), por oportunizar a realização da pesquisa.

\section{REFERÊNCIAS}

ALENCAR, F.V.; MENDES, F.A.; BARRETO, P.L.N. Sustentabilidade, desenvolvimento e globalização como uma construção paradigmática para o contexto jurídico. Ciência e Sustentabilidade, v.5, n.1, p.28-46, 2019. Disponível em: https://doi.org/10.33809/2447-4606.51201928-46. DOI: 10.33809/2447-4606.5120192846. Acesso em: 06/10/2020.

AQUILANI, B.; SILVESTRE, C.; IOPPOLO, G.; RUGGIERI, A. The challenging transition to bio-economies: towards a new framework integrating corporate sustainability and value co-creation. Journal of Cleaner Production, v.172, p.4001-4009, 2018. Disponível em: https://doi.org/10.1016/j.jclepro.2017.03.153. DOI: 10.1016/j.jclepro.2017.03.153. Acesso em: 06/06/2020. 
BARBA, R.Y.B.; SANTOS, N. A bioeconomia no século XXI: reflexões sobre a biotecnologia e a sustentabilidade no Brasil. Revista de Direito e Sustentabilidade, v.6, n.2, p.26-42, 2020. Disponível em: http://dx.doi.org/10.26668/IndexLawJournals/2525-9687/2020.v6i2.7023. DOI: 10.26668/IndexLawJournals/2525-9687/2020.v6i2.7023. Acesso em: 10/06/21.

BARROS, A.C.V. O ser humano globalizado e sua relação com a natureza: a pauta do desenvolvimento sustentável e o estabelecimento da bioeconomia. Brazilian Journal of Development, v.6, n.6, p.38147-38161, 2020. Disponível em: https://doi.org/10.34117/bjdv6n6-382. DOI: $10.34117 /$ bjdv6n6-382. Acesso em: 06/10/2020.

BARROS, R.N.; SANTOS, M.S.M.; CARDOSO, C.A.L.; BATISTOTE, M. A utilização de resíduos agroindustriais para a produção de bioetanol. Revista Gestão e Sustentabilidade Ambiental, v.8, n.1, p.31-43, 2019. Disponível em: http://dx.doi.org/10.19177/rgsa.v8e1201931-43. DOI: 10.19177/rgsa.v8e1201931-43. Acesso em: 06/10/2020.

BELTRAN, L.B.; JANUÁRIO, E.F.D.; ARAÚJO, L.A.; SOUZA, A.C.; MELONI, C.E.P. et al. Processo de biossorção de corante utilizando casca de tangerina (Citrus reticulata). Brazilian Journal of Development, v.6, n.6, p.41760-41771, 2020. Disponível em: https://doi.org/10.34117/bjdv6n6-641. DOI: 10.34117/bjdv6n6-641. Acesso em: 08/05/2021.

BIBER-FREUDENBERGER, L.; BASUKALA, A.K.; BRUCKNER, M.; BÖRNER, J. Sustainability performance of national bio-economies. Sustainability, v.10, n.8, 2705, p.1-20, 2018. Disponível em: https://doi.org/10.3390/su10082705. DOI: 10.3390/su10082705 Acesso em: 06/06/2020.

CARVALHO, S.; PIRES, C.C.; WOMMER, T.P.; LOPES, J.F.; MÔNEGO, C.O. et al. Economicidade e desempenho produtivo de cordeiros confinados submetidos a dietas com resíduos agroindustriais. Ciência Animal Brasileira, v.17, n.1, p.36-44, 2016. Disponível em: https://doi.org/10.1590/1089-6891v17i119806. DOI: 10.1590/10896891v17i119806. Acesso em: 04/06/2020.

CAVALCANTE, L.V. Reestruturação produtiva e a nova geografia do coco. CampoTerritório: Revista de Geografia Agrária, v.11, n.25, p.121-148, 2016. Disponível em: https://doi.org/10.14393/RCT112506. DOI: 10.14393/RCT112506. Acesso em: 06/10/2020.

CAVALCANTE FILHO, P.G.; BUAINAIN, A.M.; BENATTI, G.S.S. A cadeia produtiva agroindustrial do biodiesel no Brasil: um estudo sobre sua estrutura e caracterização. DRd - Desenvolvimento Regional em Debate, v.9, p.772-799, 2019. Disponível em: https://doi.org/10.24302/drd.v9i0.2252. DOI: 10.24302/drd.v9i0.2252. Acesso em: 13/10/2020. 
CEBRAP - Centro Brasileiro de Análise e Planejamento. Brazil Biotech Map 2011. São Paulo, CEBRAP, 2011. Disponível em: https://cebrap.org.br/wpcontent/uploads/2017/05/Brazil-Biotec-Map-2011.pdf. Acesso em: 05/06/2020.

CNI - Confederação Nacional da Indústria. Bioeconomia: uma agenda para o Brasil. Brasília: CNI, 2013. Disponível em: https://bucket-gw-cni-static-cmssi.s3.amazonaws.com/media/filer_public/78/86/7886aeb1-57a8-4be2-9ad9f8f31b176a8f/bioeconomia_uma_agenda_para_brasil.pdf. Acesso em: 07/06/2020.

CORDEIRO, N.K.; CARDOSO, K.P.S; MATA, T.C.; BARBOSA, J.A.; GONÇALVES JúNIOR, A.C Gestão de resíduos agrícolas como forma de redução dos impactos ambientais. Revista de Ciências Ambientais, v.14, n.2, p.23-34, 2020. Disponível em: http://dx.doi.org/10.18316/rca.v14i2.5593. DOI: 10.18316/rca.v14i2.5593. Acesso em: 09/06/2021.

CRUZ, A.C.; PEREIRA, F.S.; FIGUEIREDO, V.S. Fertilizantes organominerais de resíduos do agronegócio: avaliação do potencial econômico brasileiro. Rio de Janeiro: BNDES Setorial, v.45, p.137-187, 2017. Disponível em: https://web.bndes.gov.br/bib/jspui/bitstream/1408/11814/1/BS\%2045\%20Fertilizantes\% 20organominerais\%20de\%20res\%c3\%adduos\%20\%5b...\%5d_P_BD.pdf. Acesso em: 04/06/2020.

DIAS, H.C.; REIS, A.B. Estudo da viabilidade e desenvolvimento de filmes ativos a partir de resíduos da indústria vinícola. The Journal of Engineering and Exacts Sciences, v.5, n.5, p.444-451, 2019. Disponível em: https://dx.doi.org/10.18540/jcecvl5iss5pp0444-0451. DOI: 10.18540/jcecvl5iss5pp04440451. Acesso em: 04 jun. 2020.

DIAS, R.F.; CARVALHO, C.A.A. Bioeconomia no Brasil e no mundo: panorama atual e perspectivas. Revista Virtual de Química, v.9, n.1, p.410-430, 2017. Disponível em: https://dx.doi.org/10.21577/1984-6835.20170023. DOI: 10.21577/1984-6835.20170023. Acesso em: 05/06/2020.

DINIZ, M.C.; MARTINS, M.G.; XAVIER, K.V.M.; SILVA, M.A.A.; SANTOS, E.A. Crise global coronavírus: monitoramento e impactos. Cadernos de Prospecção, v.13, n.2, ed. $\quad$ esp., $\quad$ p.359-377, $2020 . \quad$ Disponível em: https://portalseer.ufba.br/index.php/nit/article/view/35937. DOI: 10.9771/cp.v13i2\%20COVID-19.35937. Acesso em: 06/10/2020.

DOE - United States Department of Energy. USDA - United States Department of Agriculture. USEPA - United States Environmental Protection Agency. United States Department of the Interior. NSF - National Science Foundation. USDOD - United States Department of Defense. United States Department of Transportation. United States Executive Office of the President. Federal Activities Report on the Bioeconomy. 
2016. Disponível em: https://biomassboard.gov/pdfs/farb_2_18_16.pdf. Acesso em: 07/06/2016.

EL-CHICHAKLI, B.; VON BRAUN, J.; LANG, C.; BARBEN, D.; PHILIP, J. Policy: five cornerstones of a global bioeconomy. Nature, v.535, n.7611, p.221-223, 2016. Disponível em: https://doi.org/10.1038/535221a. DOI: 10.1038/535221a. Acesso em: 06/06/2020.

EPI - Environmental Performance Index. New Haven: Yale University, 2018. Disponível em: https://epi.yale.edu/downloads/epi2018policymakerssummaryv01.pdf. Acesso em: 03/06/2020.

EUROPEAN Commission. Communication from the Commission to the European Parliament, the Council, the European Economic and Social Committee and the Committee of the Regions. Strategy for "Innovating for sustainable grow: a bioeconomy for Europe". Bruxelas: European Commission, 2012. Disponível em: https://op.europa.eu/en/publication-detail/-/publication/84e7a360-6970-4cb8-939d8acbf33f0ae8/language-en. Acesso em: 07/06/2020.

FERREIRA, V.H.S. Sustentabilidade global e desenvolvimento local. Revista Maiêutica - Urbanidades, v.1, n.01, p.21-27, 2017. Disponível em: https://publicacao.uniasselvi.com.br/index.php/URB/article/view/1685/799. Acesso em: 26/07/2020.

FRANÇA. Une stratégie bioéconomie pour la France: enjeux et vision. 2017. Disponível em: http://www1.montpellier.inra.fr/themaclic/publication/3241/2017-unestratgie-bioconomie-pour-la-france. Acesso em: 07/06/2020.

GEISSDOERFER, M.; SAVAGET, P.; BOCKEN, N.M.P.; HULTINK, E.J. The circular economy - a new sustainability paradigm? Journal of Cleaner Production, v.143, p.757-768, 2017. Disponível em: https://doi.org/10.1016/j.jclepro.2016.12.048. DOI: 10.1016/j.jclepro.2016.12.048. Acesso em: 03/06/2020.

GONÇALVES, JÚNIOR, A.C.; SCHWANTES, D.; CAMPAGNOLO, M.A.; DRAGUNSKI, D.C.; TARLEY, C.R.T. et al. Removal of toxic metals using endocarp of açaí berry as biosorbent. Water Science \& Technology, v.77, n.6, p.1547-1557, 2018. Disponível em: https://doi.org/10.2166/wst.2018.032. DOI: 10.2166/wst.2018.032. Acesso em: 10/06/21.

HASSAN, S.S.; WILLIAMS, G.A.; JAISWAL, A.K. Moving towards the second generation of lignocellulosic biorefineries in the EU: drivers, challenges, and opportunities. Renewable \& Sustainable Energy Reviews, v.101, p.590-599, 2019. Disponível em: https://doi.org/10.1016/j.rser.2018.11.041. DOI: 10.1016/j.rser.2018.11.041. Acesso em: 07/06/2020. 
HATFIELD-DODDS, S.; SCHANDL, H.; NEWTH, D.; OBERSTEINER, M.; CAI, Y. et al. Assessing global resource use and greenhouse emissions to 2050, with ambitious resource efficiency and climate mitigation policies. Journal of Cleaner Production, v.144, p.403-414, 2017. Disponível em: https://doi.org/10.1016/j.jclepro.2016.12.170. DOI: 10.1016/j.jclepro.2016.12.170. Acesso em: 06/06/2020.

HSU, A.; ESTY, D.C.; LEVY, M.A.; SHERBININ, A. 2016 Environmental Performance Index (EPI). New Haven: Yale University, 2016. Disponível em: https://doi.org/10.13140/RG.2.2.19868.90249. DOI: 10.13140/RG.2.2.19868.90249. Acesso em: 03/06/2020.

HÜNTTL, R.F.; BÜCHTING, A.J.; MÜLLER-RÖBER, B.; VON BRAUN, J.; BACHEM, A. et al. Bio-economy Council Report 2010: bio-economy innovation. Berlim: BÖR, 2011.

https://biooekonomierat.de/fileadmin/Publikationen/Englisch/bioeconomy_council_report 2010.pdf. Acesso em: 07/06/2020.

IBGE - Instituto Brasileiro de Geografia e Estatística. 2017. Disponível em: https://www.ibge.gov.br/estatisticas/multidominio/meio-ambiente/20207-contaseconomicas-ambientais-da-agua-brasil.html?=\&t=resultados. Acesso em: 20/01/2018.

IPEA - Instituto de Pesquisa Econômica Aplicada. Brasil 2035: cenários para o desenvolvimento. Brasília: IPEA/ASSECOR, 2017. Disponível em: https://www.ipea.gov.br/portal/images/stories/PDFs/livros/livros/170606_brasil_2035_ce narios_para_desenvolvimento.PDF. Acesso em: 06/06/2020.

JUMA, C.; KONDE, V. The new bioeconomy: industrial and environmental biotechnology in developing countries. Genebra: United Nations Conference on Trade and Development, 15-16 nov. 2001. Disponível em: https://www.innovations.harvard.edu/sites/default/files/newbioeconomy.pdf. Acesso em: 06/06/2020.

LAMPKOWSKI, J.C.R.; LAMPKOWSKI, M. Considerações sobre a bioeconomia e sua relação com os produtos bio-based. Caderno Meio Ambiente e Sustentabilidade, v.9, n.5, p.54-62, 2016. Disponível em: https://www.cadernosuninter.com/index.php/meioAmbiente/article/view/384. Acesso em: 20/02/2020.

LANDAU, E.C.; SILVA, G.A.; MOURA, L.; HIRSCH, A.; GUIMARAES, D.P. Dinâmica da produção agropecuária e da paisagem natural no Brasil nas últimas décadas: sistemas agrícolas, paisagem natural e análise integrada do espaço rural. v.4. Brasília: Embrapa, 2020. E-book. Disponível em: https://www.embrapa.br/busca-de-publicacoes//publicacao/1122551/dinamica-da-producao-agropecuaria-e-da-paisagem-natural-nobrasil-nas-ultimas-decadas-sistemas-agricolas-paisagem-natural-e-analise-integradado-espaco-rural. Acesso em: 08/05/2021. 
MARQUES, J.G.C.; PAIXÃO, S.K.S.; LYRA, M.R.C.C.; CARVALHO, R.M.C.M.O. et al. Gestão para a sustentabilidade no ambiente rural. Revista Gestão \& Sustentabilidade Ambiental, v.8, n.4, p.312-329, 2019. Disponível em: http://dx.doi.org/10.19177/rgsa.v8e42019312-329. DOI: 10.19177/rgsa.v8e42019312329. Acesso em: 08/05/2021.

MARTINEZ, D.G.; FEIDEN, A. Potencial do resíduo do processamento da mandioca para a produção de etanol de segunda geração. Revista Brasileira de Energias Renováveis, v.6, n.2, p.141-148, 2017. Disponível em: http://dx.doi.org/10.5380/rber.v6i2.45811. Acesso em: 10/06/21.

MCTIC - Ministério da Ciência, Tecnologia, Inovações e Comunicações. 17ª Semana Nacional de Ciência e Tecnologia. Bioeconomia: diversidade e riqueza para o desenvolvimento sustentável. 2019.2 Disponível em: https://snct.mctic.gov.br/bioeconomia-diversidade-e-riqueza-para-o-desenvolvientosustentavel/. Acesso em: 07/06/2020.

MIRANDA, C.A.R. Agroecología, interdisciplina y desarrollo rural sustentable. CampoTerritório: Revista de Geografia Agrária, v.13, n.29, p.271-285, 2018. Disponível em: https://doi.org/10.14393/RCT132912. DOI: 10.14393/RCT132912. Acesso em: 06/10/2020.

MORAES, S.L.; MASSOLA, C.P.; SACCOCCIO, E.M.; SILVA, D.P.; GUIMARÃES, Y.B.T. Cenário brasileiro de geração e uso da biomassa adensada. Revista IPT: Tecnologia e Inovação, v.1, n.4, p.58-73, 2017. Disponível em: http://revista.ipt.br/index.php/revistalPT/article/view/37. Acesso em: 04/06/2020.

MOREIRA, A.M. Bioeconomia: plataforma mundial de inovação e sustentabilidade nas cadeias agroindustriais. Revista Processos Químicos, v.10, n.20, p.351-353, 2016. Disponível em: https://doi.org/10.19142/rpq.v10i20.384. DOI: 10.19142/rpq.v10i20.384. Acesso em: 22/02/2020.

MORETTI, C.L. Investir em pesquisa agropecuária traz retorno para a sociedade brasileira. Revista de Política Agrícola, v.1, n.1, p.3-5, 2020. Disponível em: https://seer.sede.embrapa.br/index.php/RPA/article/view/1584/pdf. Acesso em: 06/10/2020.

MORRISON, B.; GOLDEN, J.S. An empirical analysis of the industrial bioeconomy: implications for renewable resources and the environment. BioResources, v.10, n.3, p.4411-4440, 2015. Disponível em: https://doi.org/10.15376/biores.10.3.4411-4440. DOI: 10.15376/biores.10.3.4411-4440. Acesso em: 06/06/2020.

NASCIMENTO FILHO, W.B.; FRANCO, C.R. Avaliação do potencial dos resíduos produzidos através do processamento agroindustrial no Brasil. Revista Virtual de 
Química, v.7, n.6, p.1968-1987, 2015. Disponível em: https://dx.doi.org/10.5935/19846835.20150116. DOI: 10.5935/1984-6835.20150116. Acesso em: 04/06/2020.

NGUYEN, Q.; BOWYER, J.; HOWE, J.; BRATKOVICH, S.; GROOT, H. et al. Global production of second-generation biofuels: trends and influences. Minneapolis: Dovetail Partners, Inc., $2017 . \quad$ Disponível em: https://dovetailinc.org/upload/tmp/1579558792.pdf. Acesso em: 07/06/2020.

NWACHUKWU, I.; EKWENYE, U.N.; AGOMUO, N.G.; OGBONNA, C. Bioconversion of agricultural and food processing wastes to value-added products using solid state fermentation technology. A Review. IOSR Journal of Pharmacy and Biological Sciences, v.11, n.5, ver.3, p.68-71, 2016. Disponível em: https://doi.org/10.9790/30081105036871. DOI: 10.9790/3008-1105036871. Acesso em: 04/06/2020.

OECD - Organisation for Economic Co-Operation and Development. The Bioeconomy to 2030: designing a policy agenda. 2009. Disponível em: https://www.oecd.org/futures/long-termtechnologicalsocietalchallenges/42837897.pdf. Acesso em: 06/06/2020.

OLIVEIRA, K.C.; ZANIN, V. A bioeconomia e os biocombustíveis no cenário brasileiro. Revista IPecege, v.1, n.2, p.23-43, 2015. Disponível em: https://doi.org/10.22167/r.ipecege.2015.2.23. DOI: 10.22167/r.ipecege.2015.2.23. Acesso em: 07/10/2020.

ONU - Organização das Nações Unidas. Transforming our world: the 2030 Agenda for Sustainable Development. New York, 25-27 sep. 2015. Disponível em: https://sustainabledevelopment.un.org/post2015/transformingourworld. Acesso em: 22/02/2020.

PARISI, C.; RONZON, T. A global view of bio-based industries: benchmarking and monitoring their economic importance and future developments. Seville: European Commission, 18-19 feb. 2016. Disponível em: https://publications.jrc.ec.europa.eu/repository/bitstream/JRC103038/lb-na-28376-enn.pdf. Acesso em: 07/06/2020.

PENHA, E.M.; VIANA, L.A.N.; GOTTSCHALKI, L.M.F.; TERZI, S.C.; SOUZA, E.F. Aproveitamento de resíduos da agroindústria do óleo de dendê para a produção de lipase por Aspergillus niger. Ciência Rural, v.46, n.4, p.755-761, 2016. Disponível em: https://doi.org/10.1590/0103-8478cr20131673. DOI: 10.1590/0103-8478cr20131673. Acesso em: 04/06/2020.

SANTIAGO, B.L.S.; RODRIGUES, F.A. Processamento de biomassa lignocelulósica para a produção de etanol: uma revisão. The Journal of Engineering and Exact Sciences, v.3, n.7, p.1011-1022, 2017. Disponível em: 
https://doi.org/10.18540/jcecvl3iss7pp1011-1022. DOI: 10.18540/jcecv|3iss7pp10111022. Acesso em: 10/06/21.

SANTOS, C.C.; BARBOSA, R.C.S.; LEÃO, L.L.; ARCANJO, M.C.N.; SOARES, L.J.F. et al. Aceitabilidade sensorial de barras de cereais formuladas a partir de resíduos agroindustriais. Revista Eletrônica Acervo Saúde, v.10, n.4, p.1864-1870, 2018. Disponível em: https://dx.doi.org/10.25248/REAS285_2018. DOI: 10.25248/REAS285_2018. Acesso em: 04/06/2020.

SARAIVA, B.R.; VITAL, A.C.P.; ANJO, F.A.; CESARIO, E.; MATUMOTO-PINTRO, P.T. Valorização de resíduos agroindustriais: fontes de nutrientes e compostos bioativos para a alimentação humana. Revista PubSaúde, v.1, a007, p.1-10, 2018. Disponível em: https://dx.doi.org/10.31533/pubsaude1.a007. DOI: 10.31533/pubsaude1.a007. Acesso em: 04/06/2020.

SARLET, I. W. Princípios do direito ambiental. 2.ed. São Paulo: Saraiva, 2017. 296p.

SCARLAT, N.; DALLEMAND, J-F.; MONFORTI-FERRARIO, F.; NITA, V. The role of biomass and bioenergy in a future bioeconomy: policies and facts. Environmental Development, v.15, p.3-34, 2015. Disponível em: https://doi.org/10.1016/j.envdev.2015.03.006. DOI: 10.1016/j.envdev.2015.03.006. Acesso em: 11/06/2020.

SILVA, D.B. Sustentabilidade no agronegócio: dimensões econômica, social e ambiental. Comunicação \& Mercado/UNIGRAN, v.1, n.3, p 23-34, 2012. Disponível em: $\quad$ https://www.unigran.br/dourados/mercado/paginas/arquivos/edicoes/3/3.pdf. Acesso em: 06/06/2020.

SILVA, G.F.; MEGALE, M.H.D.S. O direito ao futuro como mandamento ético: a sustentabilidade e o modelo de produção alimentar no Brasil. Revista Gestão \& Sustentabilidade Ambiental, v.9, n.esp.ll, p.811-822, 2020. Disponível em: http://dx.doi.org/10.19177/rgsa.v9e02020811-822. DOI: 10.19177/rgsa.v9e02020811822. Acesso em: 06/06/2020.

SILVA, L.M.; CARDOSO, A.L.C.; AVELAR, K.E.S. A sustentabilidade social como caminho para mitigar o cenário de distopia urbana das cidades em pandemia. Revista Augustus, v.25, n.51, p.130-149, 2020a. Disponível em: https://doi.org/10.15202/1981896.2020v25n51p130. DOI: 10.15202/1981896.2020v25n51p130. Acesso em: 06/10/2020.

SILVA, M.F.O.; PEREIRA, F.S.; MARTINS, J.V.B. A bioeconomia brasileira em números. Rio de Janeiro: BNDES Setorial, 2018. Disponível em: https://web.bndes.gov.br/bib/jspui/bitstream/1408/15383/1/BS47_Bioeconomia_FECH ADO.pdf. Acesso em: 07/06/2020. 
SILVA, M.L.T.; BRINQUES, G.B.; GURAK, P.D. Desenvolvimento e caracterização de bioplásticos de amido contendo farinha de subproduto de broto. Brazilian Journal of Food Technology, v.23, e2018326, p.1-13, 2020b. Disponível em: http://dx.doi.org/10.1590/1981-6723.32618. DOI: 10.1590/1981-6723.32618. Acesso em: 06/10/2020.

SOFFIATI, A. Breve história da globalização ocidental e seus custos ambientais. Ambientes: Revista de Geografia e Ecologia Política, v.2, n.1, p.144-173, 2020. Disponível em: https://doi.org/10.48075/amb.v2i1.24225. DOI: 10.48075/amb.v2i1.24225. Acesso em: 06/10/2020.

TORRES, D.A.P.; CAMPOS, S.K.; PONCHIO, A.P.S.; BARROS, G.S.C.; FIGUEIREDO, E.V.C et al. Viabilidade econômica da produção agropecuária no Brasil: aspectos gerais, metodologia e principais resultados. In: SANTOS, M.M.; CAMPOS, S.K.; TORRES, D.A.P.; PONCHIO, A.P.S. BARROS, G.S.C. et al. (org.). Sustentabilidade e sustentação da produção de alimentos no Brasil: o desafio da rentabilidade na produção. Brasília: Centro de Gestão e Estudos Estratégicos, 2014. v.2. cap.1. p.1147. E-book. Disponível em: https://www.cgee.org.br/documents/10182/734063/Des_Rentabilidade_Vol_II_LIVRO_ Web_13052015_10169.pdf. Acesso em: 06/06/2020.

THORSTENSEN, V.H.; MOTA, C.R. A governança mundial da sustentabilidade: o papel da OCDE. Working Paper 486, CCGI n. 10. São Paulo: Escola de Economia de São Paulo da Fundação Getúlio Vargas, 2018. Disponível em: http://hdl.handle.net/10438/24781. Acesso em: 03/06/2020.

TURINE, J.A.V.; MACEDO, M.L.R. Direitos humanos, comunidades tradicionais e biodiversidade: desafios para o desenvolvimento sustentável. Revista Direito UFMS, v.3, n.2, $\quad$ p.175-194, $2017 . \quad$ Disponível em: https://periodicos.ufms.br/index.php/revdir/article/view/5313. 10.21671/rdufms.v3i2.5313. Acesso em: 06/10/2020.

UNITED Nations. World population prospects. 2017. Disponível em: https://population.un.org/wpp/DataQuery/. Acesso em: 12/01/2018.

ZANELLA, T.P.; LAGO, S.M.S. A produção científica brasileira sobre a sustentabilidade no agronegócio: um recorte temporal entre 2005 e 2015. Organizações Rurais \& Agroindustriais, v.18, n.4, p.356-370, 2016. Disponível em: https://doi.org/10.21714/2238-68902016v18n4p356. DOI: 10.21714/223868902016v18n4p356. Acesso em: 06/10/2020. 